\title{
Inverse regulatory coordination of motility and curli-mediated adhesion in Escherichia coli
}

\author{
Christina Pesavento, Gisela Becker, Nicole Sommerfeldt, Alexandra Possling, Natalia Tschowri, \\ Anika Mehlis, and Regine Hengge ${ }^{1}$
}

Institut für Biologie-Mikrobiologie, Freie Universität Berlin, 14195 Berlin, Germany

\begin{abstract}
During the transition from post-exponential to stationary phase, Escherichia coli changes from the motile-planktonic to the adhesive-sedentary "lifestyle." We demonstrate this transition to be controlled by mutual inhibition of the FlhDC/motility and $\sigma^{\mathrm{s}}$ /adhesion control cascades at two distinct hierarchical levels. At the top level, motility gene expression and the general stress response are inversely coordinated by $\sigma^{70} / \sigma^{\mathrm{FliA}} / \sigma^{\mathrm{s}}$ competition for core RNA polymerase and the FlhDC-controlled FliZ protein acting as a $\sigma^{\mathrm{s}}$ inhibitor. At a lower level, the signaling molecule bis-( $\left.3^{\prime}-5^{\prime}\right)$-cyclic-diguanosine monophosphate (c-di-GMP) reduces flagellar activity and stimulates transcription of $\operatorname{csg} D$, which encodes an essential activator of adhesive curli fimbriae expression. This c-di-GMP is antagonistically controlled by $\sigma^{\mathrm{s}}$-regulated GGDEF proteins (mainly YegE) and YhjH, an EAL protein and c-di-GMP phosphodiesterase under FlhDC/FliA control. The switch from motility-based foraging to the general stress response and curli expression requires $\sigma^{\mathrm{s}}$-modulated down-regulation of expression of the flagellar regulatory cascade as well as proteolysis of the flagellar master regulator FlhDC. Control of YhjH by FlhDC and of YegE by $\sigma^{\text {s }}$ produces a fine-tuned checkpoint system that "unlocks" curli expression only after down-regulation of flagellar gene expression. In summary, these data reveal the logic and sequence of molecular events underlying the motile-to-adhesive "lifestyle" switch in E. coli.
\end{abstract}

[Keywords: Adhesion; c-di-GMP; flagella; GGDEF; EAL; RpoS]

Supplemental material is available at http://www.genesdev.org.

Received February 15, 2008; revised version accepted July 1, 2008.

Depending on environmental conditions, bacteria can exhibit very different "lifestyles." They can occur in a motile single-cellular or planktonic state or as sedentary cells that use adhesive fimbriae to cluster together and form biofilms on surfaces (O'Toole et al. 2000; HallStoodley et al. 2004). With Escherichia coli grown in complex medium, these states can be observed successively, with cells transiently becoming highly motile during the post-exponential growth phase (Adler and Templeton 1967; Amsler et al. 1993; Zhao et al. 2007) followed by the induction of adhesive curli fimbriae during entry into stationary phase (Olsén et al. 1989). Motility and adhesion can also be expected to be mutually exclusive-being sticky seems counterproductive for swimming around, whereas adhesion and settling down might require reduced activity of the force-generating flagellar "propellers." A convenient way to realize such an inverse coordination would be to endow the flagellar/ motility control system with at least one component

${ }^{1}$ Corresponding author.

E-MAIL Rhenggea@zedat.fu-berlin.de; FAX 49-30-838-53118.

Article is online at http://www.genesdev.org/cgi/doi/10.1101/gad.475808. that plays an inhibitory role in the adhesion control system and vice versa.

In E. coli, both motility and curli-mediated adhesion are under the control of regulatory feedforward cascades, each with a master regulator at the top, which acts as a massive environmental signal integrator. For flagellar expression and motility, this master regulator is the FlhDC complex $\left(\mathrm{FlhD}_{4} \mathrm{C}_{2}\right.$, with the flhDC operon being defined as the flagellar class 1 operon) (Liu and Matsumura 1994; Wang et al. 2006). FlhDC activates the expression of class 2 operons, which encode the inner part of the flagellum (i.e., the hook basal body that also acts as a secretion system for the outer components) as well as the flagellar $\sigma$ subunit of RNA polymerase (RNAP), FliA $\left(\sigma^{28}\right.$ or $\left.\sigma^{\mathrm{F}}\right)$, and its anti- $\sigma$ factor, FlgM. Upon secretion of FlgM, which occurs as soon as the basal body secretion system is functional, FliA is released to activate class 3 operons that encode the outer subunits of the flagellum, additional proteins required for flagellar function and chemotaxis, as well as a number of proteins of still unknown function (Chilcott and Hughes 2000; Karlinsey et al. 2000; Aldrigde et al. 2006).

Adhesive curli fimbriae, on the other hand, are ex- 
pressed during entry into stationary phase (in cells that grow below $30^{\circ} \mathrm{C}$ ). Curli fimbriae are involved in both cell-cell aggregation and surface adhesion (Barnhart and Chapman 2006). The curli control cascade is a module within the general stress response, for which the $\sigma^{\mathrm{s}}$ (RpoS) $\sigma$ subunit of RNAP acts as the master regulator (Hengge-Aronis 2000). In parallel, $\sigma^{\mathrm{S}}$-containing RNAP $\left(E \sigma^{\mathrm{S}}\right)$ activates the expression of MlrA (a MerR-like regulator) and YdaM (a GGDEF protein, see below), which, together with $\mathrm{E} \sigma^{\mathrm{S}}$ again, are essential to activate transcription of the $\operatorname{csg} D$ gene. The CsgD protein (also called AgfD in Salmonella) is an essential activator for the curli structural gene operon $(\operatorname{csg} B A C)$, and cooperates with the vegetative RNAP at the $\operatorname{csg} B$ promoter (Römling et al. 2000; Brown et al. 2001; Gerstel et al. 2003; Weber et al. 2006).

Another key player in the control of motility and curli expression-i.e., in bacterial "lifestyle" switching-is the signaling molecule bis- $\left(3^{\prime}-5^{\prime}\right)$-cyclic-diguanosine monophosphate (c-di-GMP) (for recent reviews, see Römling et al. 2005; Jenal and Malone 2006; Ryan et al. 2006; Tamayo et al. 2007). Overproduction of certain diguanylate cyclases (DGC; characterized by GGDEF domains) interferes with motility and strongly activates the expression of curli fimbriae and the biofilm matrix component cellulose in enteric bacteria, whereas overproduction of certain c-di-GMP-degrading phosphodiesterases ( $\mathrm{PDE}$; carrying EAL domains) produces the opposite phenotype. In recent mutational and biochemical analyses, specific DGCs and PDEs have been assigned antagonistic roles in the regulation of curli expression (Kader et al. 2006; Weber et al. 2006; Simm et al. 2007). For E. coli, these are YdaM (the GGDEF protein already mentioned above) and YciR (a GGDEF + EAL protein), which control $\operatorname{csg} D$ transcription (Weber et al. 2006). Another EAL protein, $\mathrm{YhjH}$, was shown to play a positive role in motility (Ko and Park 2000; Rychlik et al. 2002; Frye et al. 2006; Ryjenkov et al. 2006). However, the molecular details of c-di-GMP action in the control of curli expression and motility have not been characterized.

The questions addressed by our study are the following: Do the FlhDC/motility and $\sigma^{\mathrm{s}} /$ curli control cascades really directly communicate to inversely coordinate their activities? And, if so, what are the factors involved, and are (some of) these factors c-di-GMP control systems; i.e., GGDEF/EAL proteins? With the present study, we identified several such factors acting at different hierarchical levels of the control network established, we provide evidence that throwing the motility-to-adhesion switch requires a precise down-regulation of motility at the transcriptional, proteolytic, and protein activity levels, and we present a framework model for the logic and the sequence of molecular events underlying this phenotypic "lifestyle" change in $E$. coli during entry into stationary phase.

\section{Results}

FliZ, a protein under flagellar control, interferes with $\sigma^{S}$ activity and curli formation

Escherichia coli K-12 strains are quite heterogenous with respect to $\sigma^{\mathrm{S}}$ levels and activity (Jishage and Ishi- hama 1997), curli expression (Bokranz et al. 2005), and motility (Barker et al. 2004). The strain MC4100 used in our previous studies of $\sigma^{\mathrm{S}}$ and curli regulation is nonmotile due to a+1 frameshift mutation in the flhDC operon encoding the flagellar master regulator (Barembruch and Hengge 2007). Therefore, we chose another commonly used strain, W3110, which is flhDC $C^{+}$and exhibits wildtype motility (Supplemental Fig. S1). W3110 reaches very similar $\sigma^{\mathrm{s}}$ levels in stationary phase as MC4100 (data not shown) and also expresses the curli genes (assayed using a single-copy $\operatorname{csg} B:: 1 a c Z$ reporter fusion) under the same control of $\sigma^{\mathrm{s}}$, MlrA, YdaM, YciR, and CsgD as MC4100 (Supplemental Fig. S1). Interestingly, the control by YdaM seemed even more stringent in W3110, which was a first hint that the presence of the flagellar system may produce an inhibitory influence that has to be overcome by the complex curli control cascade.

In parallel, we also used strain MC4100, in which we complemented the chromosomal flhDC mutation by a low-copy-number plasmid (pFlhDC) that upon IPTG induction expresses FlhDC from the tac promoter $\left(\mathrm{p}_{\text {tac }}\right)$ at similar levels as W3110 (Barembruch and Hengge 2007). While this construct generated motility (Barembruch and Hengge 2007), it surprisingly eliminated curli gene expression as assayed using a $\operatorname{csg} B:: 1 a c Z$ reporter fusion (Supplemental Fig. S2A). Moreover, pFlhDC did so too when transformed into W3110 (data not shown). We reasoned that the major difference between strains with a single flhDC $C^{+}$allele in the chromosome and the pFlhDC constructs is the flhDC expression pattern during transition into stationary phase: Whereas wild-type strains seem to shut down flagellar gene expression during this time (Amsler et al. 1993; Barembruch and Hengge 2007; see below), $\mathrm{p}_{\text {tac }}$-driven flhDC continues to be expressed. This suggested that the flagellar FlhDC regulon indeed includes at least one inhibitor of curli expression, and that the strong curli-negative phenotype of the pFlhDCcarrying strain provided a screen for identifying this inhibitor genetically.

As a first step, we introduced a fliA mutation into the pFlhDC-carrying strain and observed a complete suppression of the inhibition of curli gene expression (Supplemental Fig. S2B). We concluded that the inhibitor for curli expression is either (1) FliA itself, which when remaining present in stationary phase at elevated levels may outcompete $\sigma^{\mathrm{s}}$ at the RNAP core enzyme, (2) a protein the expression of which is FliA-dependent (i.e., a class 3 flagellar gene product), or (3) a protein expressed from a gene right downstream from fliA onto which the fliA::cat insertion would exert a polar effect. A candidate for the latter was the fliZ gene, because at least fliZ seems to be part of an operon with fliA, and its molecular function has not been defined. Therefore, we introduced a fliZ mutation into the pFlhDC strain and again observed suppression; i.e., curli genes were expressed (Supplemental Fig. S2C). This result not only eliminated the first two possibilities for the identity of the inhibitor (since in this fliZ mutant, FliA is still expressed under pFlhDC control), but in addition indicated that FliZ itself is the inhibitor, as the fliZ mutation used here is a 
nonpolar in-frame deletion. Finally, we cloned fliZ alone onto the same low-copy-number $\mathrm{p}_{t a c}$ vector (pFliz). When this plasmid was introduced into MC4100, which due to its $f 1 h D C$ mutation does not express any other flagellar gene product besides this plasmid-encoded FliZ, it completely inhibited curli expression during entry into stationary phase even in the absence of inducer (Fig. 1B). We conclude that FliZ is a highly potent inhibitor of curli expression.

How does Fliz interfere with curli expression, and is this inhibition specific for curli? Along the curli control cascade, there are many potential targets and, as a first step, we tested whether the presence of the inhibitory plasmids pFlhDC and pFliZ affected the cellular levels of the essential curli activators $\sigma^{\mathrm{S}}$ and CsgD (Fig. 1A). While $\sigma^{\mathrm{s}}$ levels remained unchanged, CsgD levels were reduced by pFlhDC and virtually eliminated by pFliZ (even without inducer). Using single-copy lacZ reporter fusions, we then found that $\mathrm{pFliZ}$ reduced the expression of MlrA, YdaM, and YciR (Fig. 1B), which together and highly specifically control $\operatorname{csg} D$ transcription (Weber et al. 2006). This explained the effect on CsgD levels (Fig. 1A). In addition, as $m \operatorname{lr} A, y d a M$, and $y c i R$ are all under $\sigma^{\mathrm{S}}$ control, this finding also raised the possibility that other $\sigma^{\mathrm{s}}$-dependent genes might be affected as well and that FliZ might have a more general role than just controlling curli expression. Indeed, the expression of $\sigma^{\mathrm{S}}$ controlled genes not associated with curli expression was also reduced by pFliZ: While osmY, a directly E $\sigma^{\text {s. }}$ controlled gene, was partially affected (Fig. 1B), the expression of gadB-i.e., a gene involved in acid resistance, which is under multiple feedforward control of $\sigma^{\mathrm{S}}(\mathrm{We}-$ ber et al. 2005) in a manner analogous to $\operatorname{csg} B$-was also completely eliminated (Fig. 1B). Thus, while FliZ does not affect $\sigma^{\mathrm{s}}$ levels, it reduces $\sigma^{\mathrm{s}}$-dependent gene expression; i.e., $\sigma^{\mathrm{S}}$ activity.

A rather general effect on $\sigma^{\mathrm{s}}$-dependent gene expression was further corroborated by genome-wide transcriptional profiling of the pFliZ-carrying strain (Supplemental Table S1): The majority of FliZ-down-regulated genes were identified previously as $\sigma^{\mathrm{s}}$-controlled (Weber et al. 2005; H. Weber and R. Hengge, unpubl., for cells grown at $28^{\circ} \mathrm{C}$ ). Moreover, these FliZ-down-regulated genes showed a strong overlap with genes activated by $\mathrm{Crl}$ (Supplemental Table S1)-i.e., a factor that specifically supports $\sigma^{\mathrm{S}}$ in its competition with other $\sigma$ factors for RNAP core (Typas et al. 2007a)—suggesting a role for FliZ in $\sigma^{\mathrm{S}}$ control that is functionally antagonistic to that of Crl. Notably, by promoting the formation of E $\sigma^{\mathrm{S}}$, $\mathrm{Crl}$ plays a role in the timing of induction of $\sigma^{\mathrm{s}}$-dependent genes (in $\mathrm{crl}$ mutants, their activation during entry into stationary phase is delayed) (see Supplemental Fig. S3; Typas et al. 2007a).

What is the role of Fliz in the motility-to-adhesion transition during entry into stationary phase? The essential activating components for transcription of the curli activator CsgD—i.e., E $\sigma^{\mathrm{s}}$, YdaM, and MlrA — are induced in a reproducible temporal order: While $E \sigma^{\mathrm{s}}$-mediated expression of genes with high-affinity promoters such as osmY started as early as an $\mathrm{OD}_{578}$ of 1 , ydaM expression started around an $\mathrm{OD}_{578}$ of 2 , and $m l r A$ expression finally began only around an $\mathrm{OD}_{578}$ of 3 (Fig. 1B). We observed that the timing of induction of the "late" gene mlrA was most sensitive to FliZ (Fig. 2A): In a fliZ knockout mutant, $m \operatorname{lr} A$ expression started more than an hour earlier (at an $\mathrm{OD}_{578}$ even below 2 already) (for comparison, see effects on mlrA expression of mutations that affect $E \sigma^{\mathrm{S}}$ levels such as $c r l$ in Supplemental Fig. S3). Also, the expression of ydaM reproducibly started somewhat earlier in the fliZ mutant (Fig. 2B). In order to show that fliZ affects $\sigma^{\mathrm{S}}$ activity and not some other factor possibly involved in the control of these genes, we also tested a synthetic $\sigma^{\mathrm{s}}$-dependent promoter (synP9, present in a single-copy lacZ fusion), which does not respond to any other regulatory factors besides $\sigma^{\mathrm{S}}$, and is sensitive even to gradual variations in the cellular content of $\sigma^{\mathrm{s}}$-containing RNAP (Typas et al. 2007a). In addition, synP9 is a typical $\sigma^{\mathrm{S}}$-dependent promoter without -35 and with $\sigma^{\mathrm{S}}$-specifically extended -10 regions (Typas et al. 2007b) (the mlrA and ydaM promoter regions are of the same type) (our unpublished data). Stationary phase induction of the synP9::1acZ fusion also started earlier in the fliZ mutant (Fig. 2C).

Taken together, these data indicate that during the post-exponential phase, FliZ transiently interferes with $\sigma^{\mathrm{S}}$ activity; i.e., either with $\mathrm{E} \sigma^{\mathrm{S}}$ formation or with $\mathrm{E} \sigma^{\mathrm{S}}$ activity directly at $\sigma^{\mathrm{S}}$-controlled promoters. Thereby, FliZ can act as a timing device for curli expression, since among the essential activators of $\operatorname{csg} D$ transcription, MlrA exhibits the latest expression and is most delayed by Fliz.

\section{Down-regulation of motility by $a \sigma^{S}$-controlled c-di-GMP module comprising the GGDEF proteins YegE/YedQ/Yeal and the EAL protein YhjH}

With FliZ, the flagellar system contains a factor that during the post-exponential growth phase interferes with the general stress response and therefore also with adhesive curli fimbriae expression. In addition, there is another "promotility" factor-i.e., the EAL protein YhjHthat is expressed from a completely FliA-dependent class 3 gene (Fig. 4D, below; Frye et al. 2006). Overexpression of YhjH could rescue motility of an otherwise nonmotile hns mutant (Ko and Park 2000). The E. coli yhjH mutant exhibited strongly reduced motility on soft agar plates (Fig. 3A). Purified YhjH was active as a c-di-GMP-cleaving phosphodiesterase (Fig. 3B), and the glutamic acid in its ELL sequence (corresponding to the generic EAL motif in this protein family) was essential for its enzymatic activity in vitro (Fig. 3B) as well as for its biological activity in vivo (i.e., for complementation of a yhjH mutation; data not shown). Both in Salmonella and E. coli, the effects of the $y$ hiH mutation on motility depend on the presence of the YcgR protein (Fig. 3A; Ryjenkov et al. 2006; Girgis et al. 2007), which is also under flagellar control (Frye et al. 2006) and belongs to the family of PilZ proteins that function as c-di-GMP-binding effector proteins (Ryjenkov et al. 2006; Benach et al. 2007; Christen et al. 2007). Reduced motility in liquid cultures 

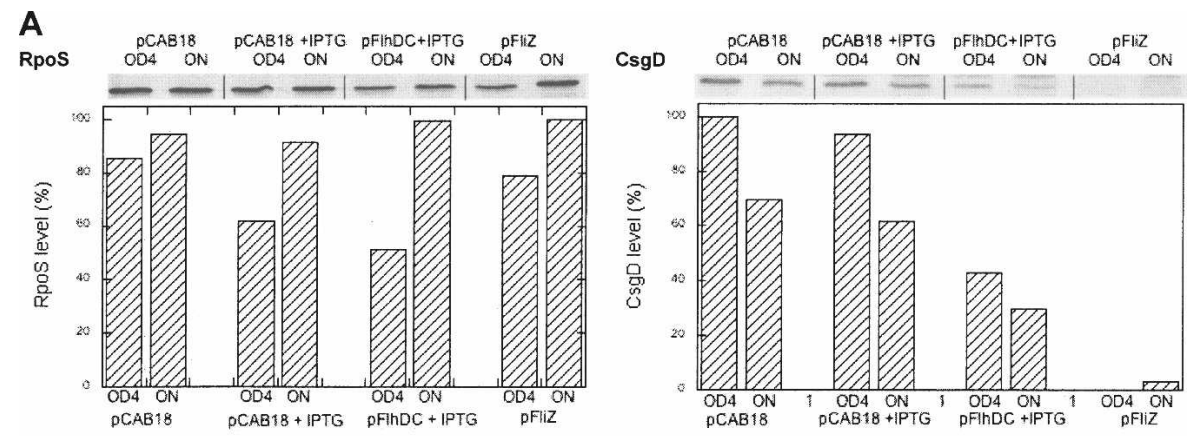

B
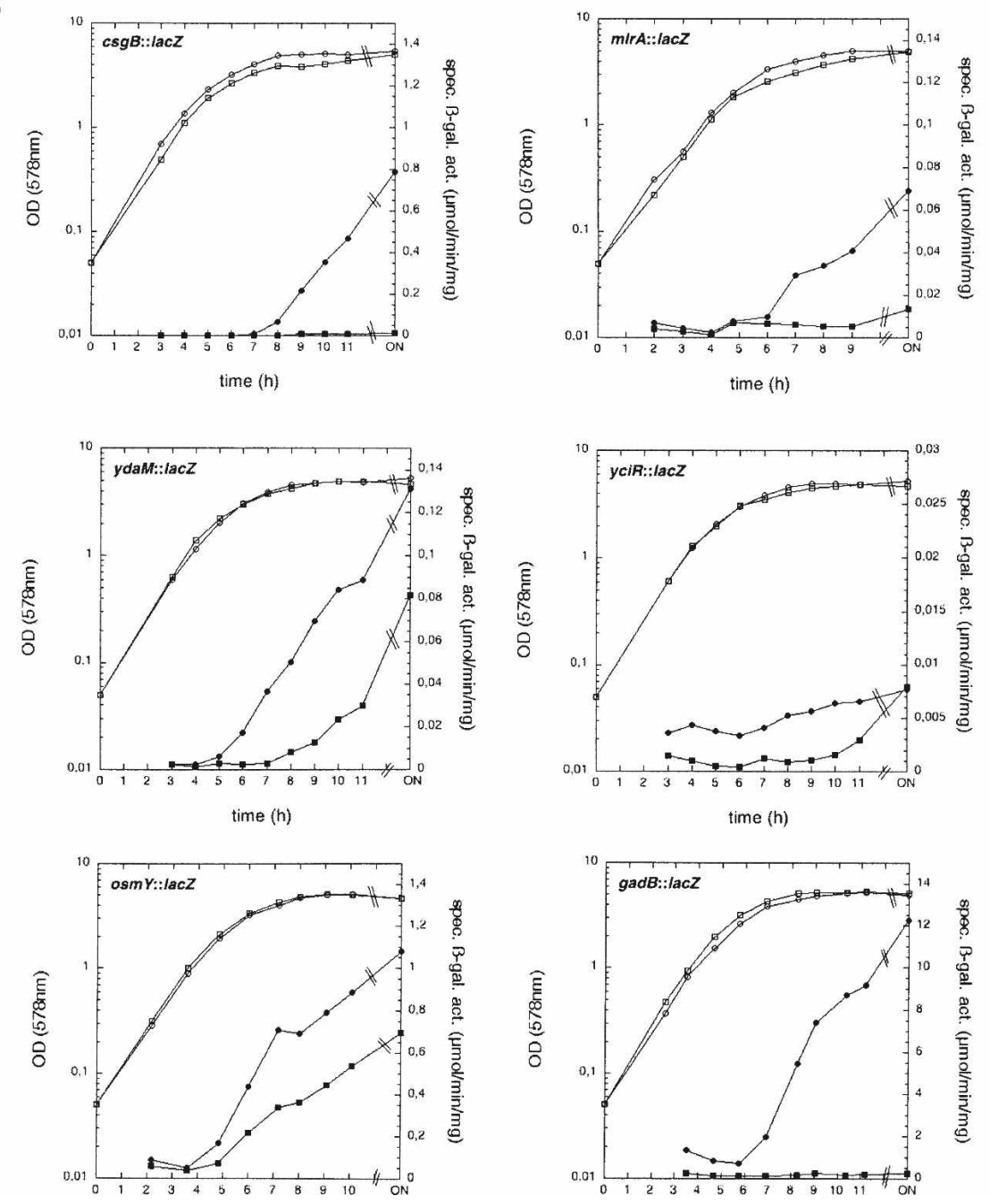

Figure 1. FliZ is an inhibitor of $\sigma^{\mathrm{s}}$ activity. (A) FliZ does not affect $\sigma^{\mathrm{s}}$ levels, but strongly down-regulates the $\sigma^{\mathrm{s}}$-controlled curli regulator CsgD. Derivatives of strain MC4100 carrying either pFlhDC, pFliZ, or the vector (pCAB18) alone were grown in the presence or absence of IPTG as indicated. Samples were taken for immunoblot analysis of $\sigma^{\mathrm{S}}$ and $\mathrm{CsgD}$ at an $\mathrm{OD}_{578}$ of 4.0 and after overnight growth $(\mathrm{ON})$. Densitometric quantification is shown below the blots. $(B)$ FliZ reduces the expression of $\sigma^{\mathrm{S}}$-controlled genes. Expression patterns of the $\sigma^{\mathrm{s}}$-dependent genes $\operatorname{csg} B, \operatorname{mlr} A, y d a M, y c i R, o s m Y$, and $\operatorname{gadB}$ were recorded using appropriate single-copy reporter fusions in strain MC4100 carrying either pFliZ (squares) or the vector (pCAB18; circles). Cells were grown without IPTG (as even low levels of FliZ are sufficient to interfere with the expression of the genes shown), and $\mathrm{OD}_{578}$ (open symbols) and specific $\beta$-galactosidase activities (closed symbols) were determined along the growth curve.

could actually be observed directly under the microscope. While all strains tested finally became nonmotile in stationary phase (probably due to reduced energy sup- ply), the $y h j H$ mutant began to reduce speed in the swimming phase at an $\mathrm{OD}_{578}$ of 3.5-3.6 already, followed by the parental strain (W3110) about an hour later 


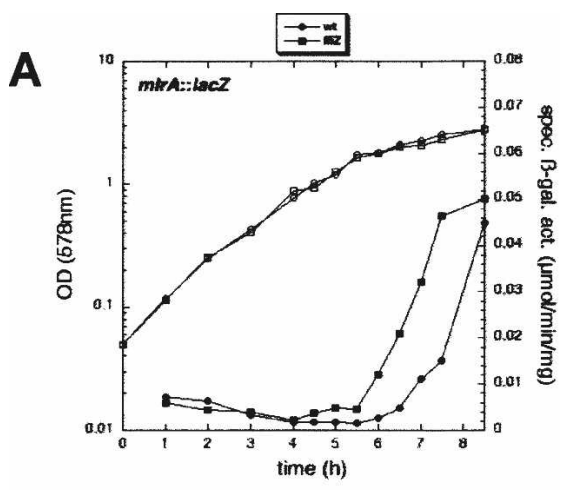

B

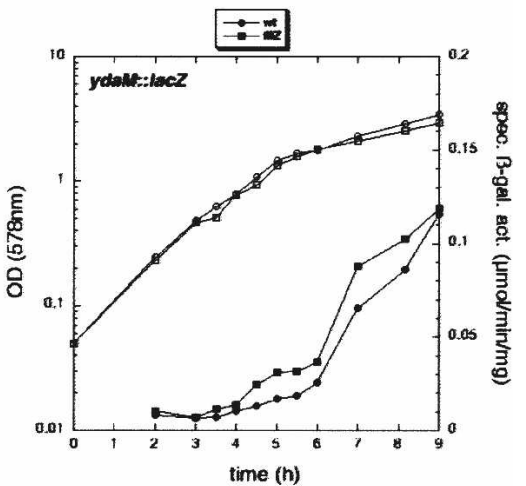

C

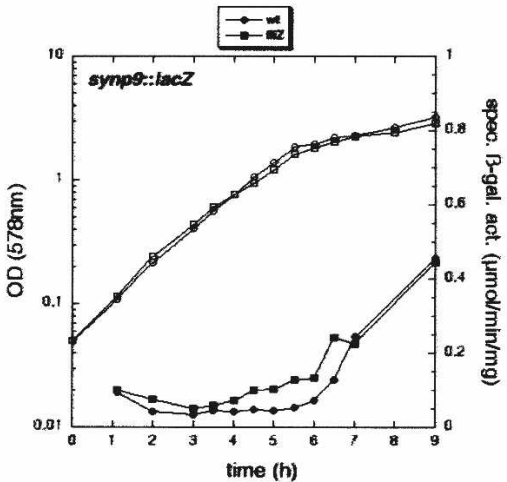

Figure 2. Mutations in $f l i Z$ affect the timing of induction of $\sigma^{\mathrm{S}}$-dependent genes. Two directly $\sigma^{\mathrm{S}}$-controlled genes encoding MlrA $(A)$ and YdaM $(B)$ (i.e., the factors essential for the expression of the curli activator CsgD), as well as a construct with a synthetic promoter that responds exclusively to $\sigma^{\text {s }}$, synp9 $(C)$, were assayed. Derivatives of strain W3110 carrying single-copy lacZ reporter fusions to $m \operatorname{lr} A, y d a M$, and synp9 and the corresponding fliZ knockout mutants were grown in LB (for symbols, see the figure). $\mathrm{OD}_{578}$ (open symbols) and specific $\beta$-galactosidase activities (closed symbols) were determined.

(at an $\mathrm{OD}_{578}$ of 3.7-3.8), whereas the $y c g R$ mutant began to reduce swimming speed only at an $\mathrm{OD}_{578}$ beyond 4.0; i.e. $>2 \mathrm{~h}$ later than the parental strain (data not shown).

The di-guanylate cyclase(s)-i.e., the GGDEF protein(s) - that provide the c-di-GMP necessary to reduce motility have not been characterized, but a previously observed partial suppression of the motility impairment of a yhjH mutant by a yegE mutation suggested that the
GGDEF+EAL protein YegE may be involved (Girgis et al. 2007). Here, we tested whether knockout mutations in any of the 19 GGDEF domain-encoding genes could suppress the nonmotile phenotype of the yhjH mutant. When cells were grown at $28^{\circ} \mathrm{C}$, two mutations, yegE and to a minor extent yedQ, were found to be partially effective (Fig. 3A). The yegE yedQ double knockout completely restored motility of the $y h j H$ mutant, indicating that the two proteins function in a redundant and additive manner, with YegE being the major player. At $37^{\circ} \mathrm{C}$ (where curli fimbriae are not expressed), another GGDEF gene, yeaI, exhibited most pronounced suppression when knocked out alone, and in order to obtain wildtype motility, yeaJ, yegE, and yedQ had to be mutated all
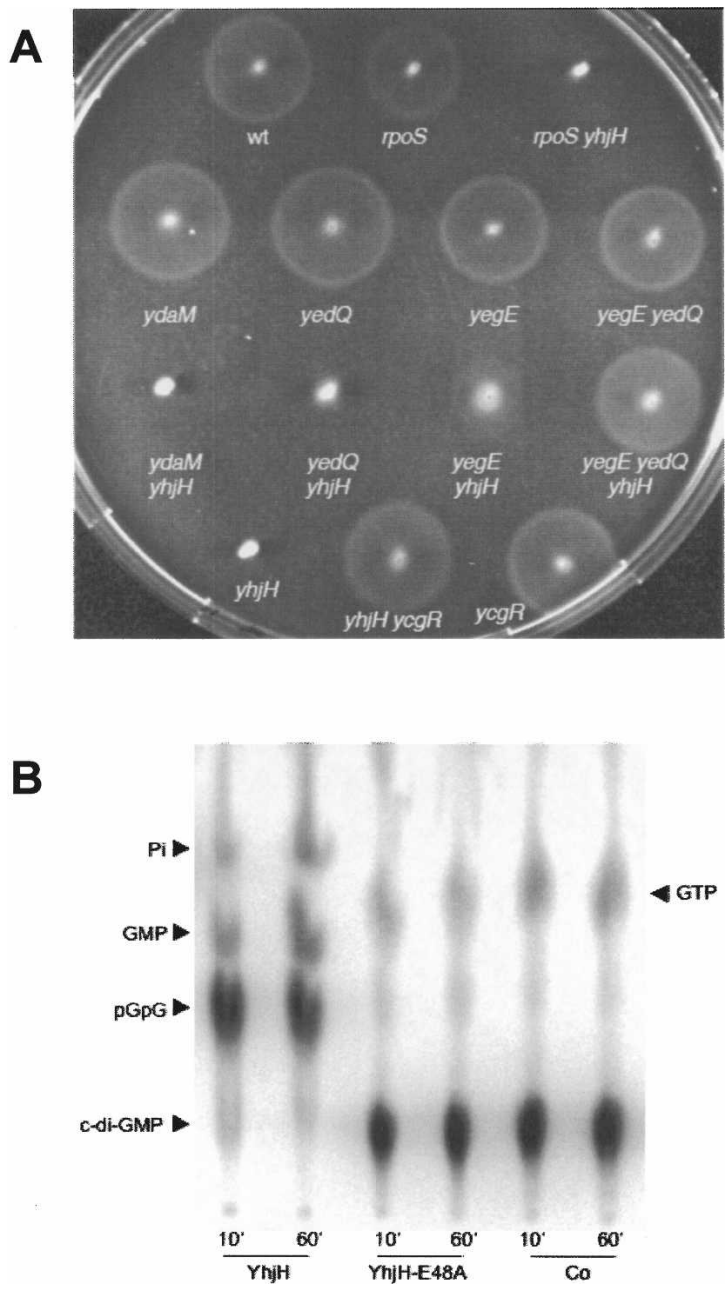

Figure 3. A complex c-di-GMP control module interferes with motility. (A) Knockout mutations in the genes for the GGDEF proteins YegE and YedQ (but not YdaM) and the c-di-GMP effector YcgR suppress the nonmotile phenotype of strains defective for the EAL domain protein $\mathrm{YhjH}$. Single, double, and triple mutants defective in the genes indicated were tested for motility on swim plates incubated at $28^{\circ} \mathrm{C}$. (B) YhjH is a c-di-GMP phosphodiesterase (PDE). Purified YhjH [whose EAL ${ }^{(48-50)}$ domain actually features an ELL sequence] and $\mathrm{YhjH}^{\mathrm{E} 48 \mathrm{~A}}$ were tested for PDE activity in vitro using radiolabeled c-di-GMP. Cleavage products are indicated. 
together (Supplemental Fig. S4). These data indicate that, depending on growth temperature, YegE $\left(28^{\circ} \mathrm{C}\right)$ or YeaJ $\left(37^{\circ} \mathrm{C}\right)$, and to a minor extent, YedQ, are the GGDEF proteins that provide the c-di-GMP that via YcgR reduces motility.

Notably, the GGDEF protein YdaM, which is the di-
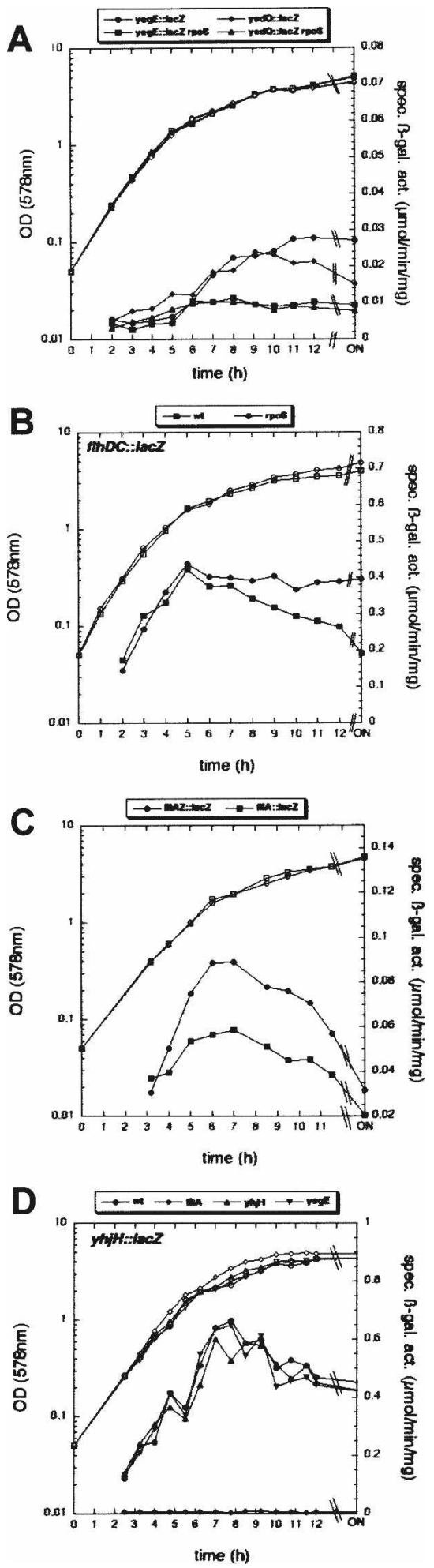

guanylate cyclase absolutely required for curli expression (see Fig. 6C, below; Weber et al. 2006), does not contribute to motility control, as its knockout mutation did not produce even traces of suppression of the yhjH nonmotility phenotype (Fig. 3A; it should be noted that the ydaM gene is induced already at an $\mathrm{OD}_{578}$ of 0.3 in the tryptone medium used for motility plates; data not shown). In addition, eliminating the EAL protein and phosphodiesterase YciR, which acts as an antagonist to YdaM in curli control (Weber et al. 2006), had no effect at all on motility (Supplemental Fig. S5); i.e., its presence in the yhiH mutant cannot compensate for the absence of YhjH. Thus, the YdaM/YciR c-di-GMP control module does not "cross-talk" into motility regulation.

We then tested whether the GGDEF proteins YegE and YedQ that down-regulate motility might somehow be linked to the $\sigma^{\mathrm{S}} /$ curli control cascade. Using single-copy $1 a c Z$ reporter fusions, we observed that both yegE and yedQ exhibited low basal expression during log phase that increases during transition into stationary phase, and that this induction was dependent on $\sigma^{\mathrm{S}}$ (Fig. 4A). Thus, by stimulating the expression of the GGDEF proteins YegE and YedQ, $\sigma^{\mathrm{S}}$ seems to positively contribute to the cellular c-di-GMP balance.

\section{Switching from motility to adhesion requires down-regulation of expression of the flagellar system}

Our finding that continued ectopic expression of the flagellar master regulator FlhDC under $\mathrm{p}_{\text {tac }}$ control during entry into stationary phase strongly inhibited curli expression (Supplemental Fig. S2) indicated that in a wildtype strain, down-regulation of flagellar gene expression is a prerequisite for the activation of the curli genes. In order to monitor such a physiological stop of expression for all relevant regulatory proteins at the different hierarchy levels, we used the single-copy reporter fusions flhDC::lacZ, fliA::lacZ, fliAZ::lacZ, and yhjH::lacZ (the latter also represents class 3 expression in general). Their expression patterns (Fig. 4B-D) demonstrated that all these genes (and therefore most likely the entire flagellar system) were activated during the early post-exponential phase. Then, however, expression of these genes stopped at an $\mathrm{OD}_{578}$ between 2 and 2.5, with the class 3 gene $y h j H$ reproducibly shutting down later than the class 1 and 2 operons. As flhDC down-regulation was less pronounced in a rpoS mutant (Fig. 4B), $\sigma^{\mathrm{S}}$, which is induced during this phase of the growth cycle, directly or indirectly contributed to this stop of expression of flagellar genes.

Figure 4. Expression patterns of genes relevant for the motility-to-adhesion switch. Derivatives of strain W3110 carrying single-copy lacZ reporter fusions in yegE and yedQ $(A)$, flhDC $(B$; fusion inserted early in $f l h C)$, fliA and fliAZ $(C$; the latter fusion inserted early in $f(i Z)$, and $y h j H(D)$ as well as knockout mutations in the genes indicated in the boxes above the single graphs, were used for the determination of $\mathrm{OD}_{578}$ (open symbols) and specific $\beta$-galactosidase activities (closed symbols) along the growth curve. 
We then wondered whether this stop of expression of the flagellar system might also be linked to the activity of the YegE+YedQ/YhjH/YcgR system, which reduces motility as described above. However, neither the yegE nor the $y h j H$ knockout mutations had any effect on the expression of the yhjH::lacZ fusion (which is used here as a representative for the overall class 3 output of the flagellar control system; Fig. 4D). Additional class 2 and 3 reporter fusions tested (flgA::1acZ and $f \lg M:: 1 a c Z$, respectively) were also not affected (data not shown).

We conclude that the reduction of motility during entry into stationary phase is based on two separate components: (1) a shut-down of flagellar gene expression during entry into stationary phase, which is required for curli expression to occur, and which is triggered by signals that act independently from c-di-GMP; and (2) a reduction of flagellar activity; i.e., altered motor function and reduced swimming speed, which is modulated by the c-di-GMP control module consisting of YegE+YedQ/YhjH/YcgR.

Switching from motility to adhesion requires ClpXP-mediated proteolysis

Although the shut-down of de novo expression of flagellar proteins is necessary for switching from motility to curli-mediated adhesion during entry into stationary phase, it may actually not be sufficient, since the already present "promotility" regulatory proteins may not be sufficiently diluted by future cell divisions any more (after shut-down of flagellar gene expression at an $\mathrm{OD}_{578}$ of 2.5 , the remaining culture cell mass increases until stationary phase just twofold). Thus, these regulators should be inactivated or even proteolytically degraded. For the master regulator of flagellar gene expression-i.e. the FlhDC complex-rapid proteolysis has indeed been shown already (Tomoyasu et al. 2003), although the physiological function of FlhDC degradation has remained unknown.

Here, we observed that a $c l p P$ mutant, which lacks the proteolytic subunit of the ClpXP and ClpAP proteases, was completely defective for curli expression (Fig. 5A)even though $\sigma^{\mathrm{S}}$-i.e., the master regulator in the curli control cascade-is itself a ClpXP substrate and therefore accumulates earlier and in higher amounts in the clpP mutant (Hengge-Aronis 2002). The inability of the clpP mutant to express curli was completely suppressed by the flhDC knockout mutation (Fig. 5A), indicating that the interference of the flagellar system with curli expression was responsible for this phenotype. The relevant component, however, was not FliZ, as a fliZ mutation did not suppress the curli-negative phenotype of the $\operatorname{clpP}$ mutant (Fig. 5A). In contrast, knocking out fliA (no matter whether polar on fliZ or not; data not shown) and yhjH (Fig. 5A) resulted in complete suppression. This suggested that, with respect to curli expression, the problem in the $\operatorname{clp} P$ mutant is a failure to down-regulate YhjH appropriately. Moreover, it also indicated that c-di-GMP controlled by $\mathrm{YhjH}$ not only reduces motility
A

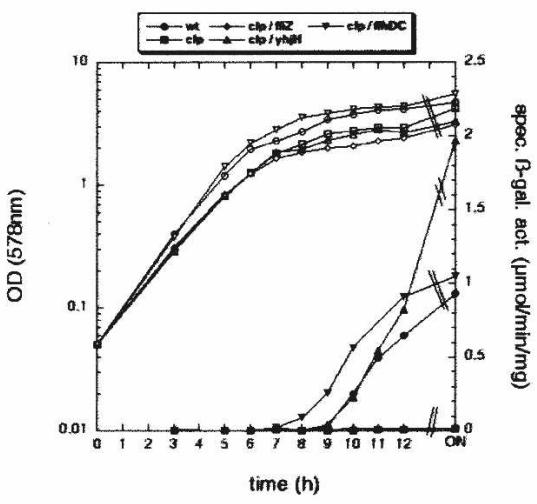

B

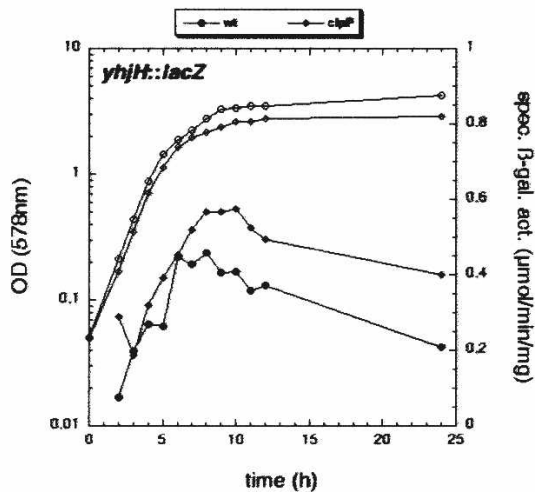

Figure 5. The Clp protease plays a key role in down-regulating $\mathrm{YhjH}$, which is a prerequisite for inducing curli expression during entry into stationary phase. $(A)$ A clpP derivative of W3110 (carrying $\operatorname{csg} B:: 1 a c Z$ as a reporter) does not induce curli expression, and this defect is suppressed by secondary mutations in flhDC and yhjH, but not in fliZ (for symbols, see figure). (B) Under the same conditions, the expression of a single-copy yhjH::lacZ fusion was tested in $c l p P^{+}$and $c l p P$ mutant backgrounds. $\mathrm{OD}_{578}$ (open symbols) and specific $\beta$-galactosidase activities (closed symbols) were determined along the growth curve of LB-grown cells.

but also has a positive influence on curli expression (see further below).

A failure of the $\operatorname{clpP}$ mutant to appropriately downregulate $\mathrm{YhjH}$ could mean that either $\mathrm{YhjH}$ is itself a Clp protease substrate, or that prolonged presence of the flagellar master activator FlhDC, a known ClpXP substrate, may result in a failure to shut down the expression of the yhjH gene in due time. The second possibility seems correct, as $\mathrm{YhjH}$ protein levels were only slightly reduced after the stop of expression around an $\mathrm{OD}_{578}$ of 2.5 (consistent with dilution by still ongoing cell division) (Supplemental Fig. S6A), YhjH did not rapidly disappear upon spectinomycin-induced cessation of total protein synthesis (Supplemental Fig. S6B), and the $c l p P$ mutation did not aggravate the partial down-regulation of curli expression by low-level ectopic $\mathrm{YhjH}$ expression (i.e., uncoupled from its natural control by FlhDC) (Supplemental Fig. S6C). On the other hand, the expression of yhjH in the $c l p P$ mutant was prolonged by $\sim 2 \mathrm{~h}$ during entry 
into stationary phase (Fig. 5B). This indicated that a precise shut-down of $\mathrm{YhjH}$ expression around an $\mathrm{OD}_{578}$ of 2.5 in a wild-type strain requires FlhDC proteolysis and is essential for the ability of the concomitantly induced GGDEF proteins YegE/YedQ to overcome the PDE activity of $\mathrm{YhjH}$ and thereby shift the c-di-GMP balance as required for the induction of curli.

The GGDEF/EAL proteins YegE and YhjH connect inhibition of motility to activation of curli expression

The involvement of $\mathrm{YhjH}$ in curli repression in the $c l p P$ mutant suggested that the entire YegE+YedQ/YhjH/ YcgR c-di-GMP control module may not only regulate motility, but also curli expression. Indeed, while the yegE mutation reduced curli expression by $\sim 60 \%$, the yhjH mutation increased curli expression (Fig. 6A). The yedQ mutation had no effect (also when introduced in addition to the yegE mutation; data not shown). Strikingly, however, YcgR is not the c-di-GMP-binding effector responsible for YegE-mediated activation of curli expression, since the $y c g R$ mutation even slightly increased curli expression (Fig. 6B). Moreover, increased curli expression in the $y$ hjH mutant was not suppressed by the $y c g R$ mutation (Fig. 6B), in contrast to the nonmotile phenotype of the yhjH mutant (Fig. 3A). We conclude that YegE/YhjH-controlled c-di-GMP activates curli expression, but that it does so not via the effector protein YcgR. Thus, YegE/YhjH-controlled c-di-GMP affects different processes via different effector components.

Finally, we wanted to know at which level of the $\sigma^{\mathrm{s}} /$ curli regulatory cascade YegE/YhjH-controlled c-di-GMP comes into play. The yegE and yhjH mutations did not affect $\sigma^{\mathrm{S}}$ levels, but did alter CsgD levels (Fig. 6D). In addition, the $y e g E$ and $y h j H$ mutations did not change the expression of the regulators of $c s g D$ transcription, i.e., YdaM, YciR, and MlrA (assayed with their respective lacZ fusions) (Supplemental Fig. S7); i.e., YegE and $\mathrm{YhjH}$ do not have a general influence on $\sigma^{\mathrm{s}}$ activity and therefore the general stress response. However, YegE/ YhjH did affect $\operatorname{csg} D$ mRNA levels (Fig. 6E). As ectopic expression of CsgD from exactly the same $\operatorname{csg} D$ mRNA transcribed from the $\mathrm{p}_{\mathrm{BAD}}$ promoter was not altered by the yegE and yhjH mutations (Supplemental Fig. S8), we conclude that the $\mathrm{YegE} / \mathrm{YhjH}$ system controls $\operatorname{csg} D$ transcription rather than $\operatorname{csg} D$ mRNA degradation, and by affecting CsgD levels, regulates curli expression.

\section{Discussion}

Switching from motility to adhesion is based on mutual inhibition of the flagellar and curli control cascades

The natural change from the motile-planktonic to the adhesive-sedentary state during transition from the postexponential to the stationary phase of growth of $E$. coli provides a convenient model system for studying a bacterial "lifestyle" switch. It should be noted, however, that "adhesion" or "sedentary state" are not semantically exchangeable with the term "biofilm." Rather, in a developing biofilm, motile and sedentary subpopulations can coexist (Klausen et al. 2006), and it may be that the molecular switch mechanisms described here, which produce a temporal succession of the motile and adhesive states in a liquid culture, are also involved in the differentiation into spatially separated motile and sessile subpopulations in a growing biofilm.

We show here that the two complex transcription fac-
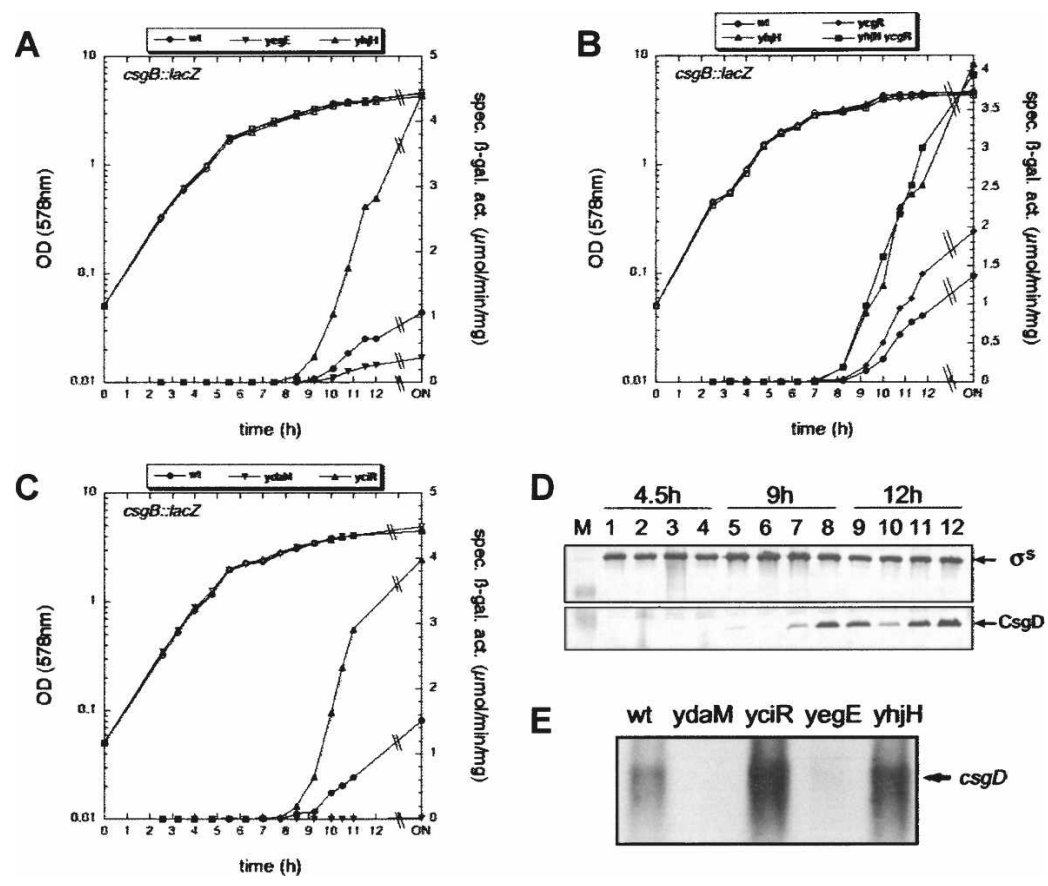

time (h)

D

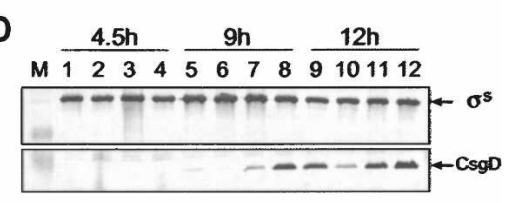

E

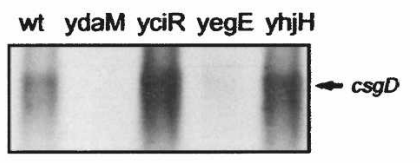

Figure 6. The GGDEF/EAL proteins YegE and YhjH affect curli expression by controlling $\operatorname{csg} D$ transcription, but in this process do not act via the c-di-GMP-binding protein YcgR. (A-C) $\operatorname{csg} B:: 1 a c Z$ expression was assayed in derivatives of strain W3110 carrying mutations in yegE, $y h j H, y c g R, y d a M$, or yciR as indicated (for symbols, see figure). $\mathrm{OD}_{578}$ (open symbols) and specific $\beta$-galactosidase activities (closed symbols) were determined along the growth curve. $(D) \sigma^{\mathrm{s}}$ and CsgD levels were determined by immunblotting of strain W3110 (lanes 1,5,9) and its derivatives with mutations in yegE (lanes 2,6,10), yegE and $y h j H$ (lanes 3,7,11), and yhjH (lanes 4,8,12). Samples were taken $4.5,9$, and $12 \mathrm{~h}$ after inocculation (corresponding to an $\mathrm{OD}_{578}$ of 1.0, 3.0, and 4.0, respectively). (E) $\operatorname{csg} D$ mRNA levels were determined by Northern analysis in W3110 and its mutant derivatives as indicated, with samples taken at an $\mathrm{OD}_{578}$ of 4.0 . 
tor cascades in E. coli that control expression and function of the motility system, and the expression of the adhesive curli fimbriae, respectively, operate in a mutually exclusive way by negatively "cross-talking" at different hierarchical levels (summarized in Fig. 7). The FlhDC/motility control system contains at least three components that down-regulate the $\sigma^{\mathrm{S}} /$ curli control system (Fig. 7, red arrows): (1) FliZ interferes with $\sigma^{\mathrm{s}}$ activity, (2) FliA $\left(\sigma^{\mathrm{F}}\right)$ competes with $\sigma^{\mathrm{S}}$ for RNAP core enzyme, and $(3)$ the EAL protein and phosphodiesterase $\mathrm{YhjH}$ eliminates c-di-GMP that otherwise (via YcgR) inhibits motility and (via a second still unknown effector component) activates $\operatorname{csg} D$ transcription and therefore curli expression. On the other hand, $\sigma^{\mathrm{s}}$, which is strongly upregulated during entry into stationary phase, contributes to down-regulation of motility (Fig. 7, green arrows) (1) by increasingly competing with $\sigma^{70}$ and FliA for RNAP core enzyme, and (2) by inducing the GGDEF proteins YegE and YedQ that by producing c-di-GMP counteract the activity of $\mathrm{YhjH}$.

This complex mutual inhibition of the two control cascades provides the basis of a switch mechanism. Throwing the switch from motility to adhesion during transition into stationary phase requires not only $\sigma^{\mathrm{s}}$ accumulation but also a precisely timed shut-off of flagellar gene expression, for which a cessation of $f 1 h D C$ expression as well as the ClpXP-mediated proteolysis of the motility master regulator FlhDC are essential. Only then $\mathrm{YhjH}$ is reduced sufficiently that its c-di-GMP-reducing PDE activity can be overcome by the increasingly expressed GGDEF proteins YegE and YedQ (Fig. 7).

With FliZ, the flagellar system contains a potent inhibitor for the general stress response and curli expression

FliZ is encoded in a flagellar class $2 / 3$ operon together with the flagellar $\sigma$ factor FliA, but FliZ is not required for motility (a fliZ derivative of W3110 is even slightly more motile than the parental strain; data not shown), and also in Salmonella a fliZ mutation had a minor modulatory effect only (Kutsukake et al. 1999) or even no effect (Frye et al. 2006) on flagellar gene expression. Here, we show that FliZ is a potent inhibitor for the $\sigma^{\mathrm{S}}$-controlled general stress response (Fig. 1; Supplemental Table S1), with those genes being most affected that are under multiple $\sigma^{\mathrm{s}}$ control in feedforward loops $(\operatorname{csg} B$, $g a d B$ ). As $\sigma^{\mathrm{s}}$ levels are not affected (Fig. 1), FliZ reduces $\sigma^{\mathrm{S}}$ activity. Interestingly, there is a strong overlap between FliZ-down-regulated and Crl-activated genes (Supplemental Table S1). Crl binds to $\sigma^{\mathrm{s}}$ (Bougdour et al. 2004) and thereby stimulates E $\sigma^{\mathrm{S}}$ holoenzyme formation during entry into stationary phase (Typas et al. 2007a). During entry into stationary phase, a crl mutant exhibits a delay in the expression of certain $\sigma^{\mathrm{S}}$-dependent genes, which have relatively low-affinity promoters and are therefore most susceptible to lower cellular levels of $\mathrm{E}^{\mathrm{S}}$ (Typas et al. 2007a).

Formally, Fliz could be an anti- $\sigma$ factor for $\sigma^{\mathrm{S}}$. Alternatively, it may modulate the activity of $E \sigma^{\mathrm{S}}$ at a subset of promoters (e.g., those with low affinity). Both mechanisms would be consistent with FliZ apparently antagonizing Crl. A simple anti- $\sigma^{\mathrm{s}}$ activity, however, seems unlikely as overproduction of FliZ is detrimental for growth also in rapidly growing cells in which $\sigma^{\mathrm{S}}$ is hardly present (data not shown); even total inhibition of $\sigma^{\mathrm{S}}$ activity would not be expected to inhibit growth (as rpoS knockout mutations do not have such impact). Moreover, FliZ contains a region with similarity to certain phage integrases, which is also present in XerD, a DNAbinding protein and site-specific recombinase involved in chromosome decatenation (Subramanya et al. 1997).

While the precise biochemical function of FliZ remains to be characterized, the physiological function of FliZ is already apparent: By interfering with the activity of $\sigma^{\mathrm{S}}$, which already accumulates during the post-expo-
Figure 7. A model summarizing the communication network between the FlhDC/motility and $\sigma^{\mathrm{S}} /$ curli regulatory cascades and the role of different c-di-GMP control modules. At the top of the network, motility and the entire general stress response are inversely coordinated by competition of $\sigma^{70}, \sigma^{\mathrm{FliA}}$, and $\sigma^{\mathrm{S}}$ for RNAP core, with FliZ being a "promotility" component that interferes with $\sigma^{\mathrm{s}}$ activity. At lower levels of the network hierarchy, motility and adhesion (via curli fimbriae) are inversely coordinated by two separately acting c-di-GMP control modules involving GGDEF/EAL proteins: YegE(+YedQ)/YhjH down-regulates flagellar activity and positively modulates $\operatorname{csg} D$ transcription, whereas YdaM/YciR acts specifically in and is essential for $\operatorname{csg} D$ transcription only (for more details, see Discussion). Also included is a third GGDEF/EAL module (i.e., YaiC/YoaD)

that is expressed under $\sigma^{\mathrm{s}}$ control later during entry into stationary phase (Weber et al. 2006; our unpublished data) and regulates the activity of cellulose synthase (Römling et al. 2005; Brombacher et al. 2006). Negative effects of the FlhDC/motility system onto the $\sigma^{\mathrm{s}} /$ curli system are highlighted in red, effects by which the $\sigma^{\mathrm{s}} /$ curli system down-regulates the FlhDC/motility system are in green. Triangles indicate additional signal input not further specified.

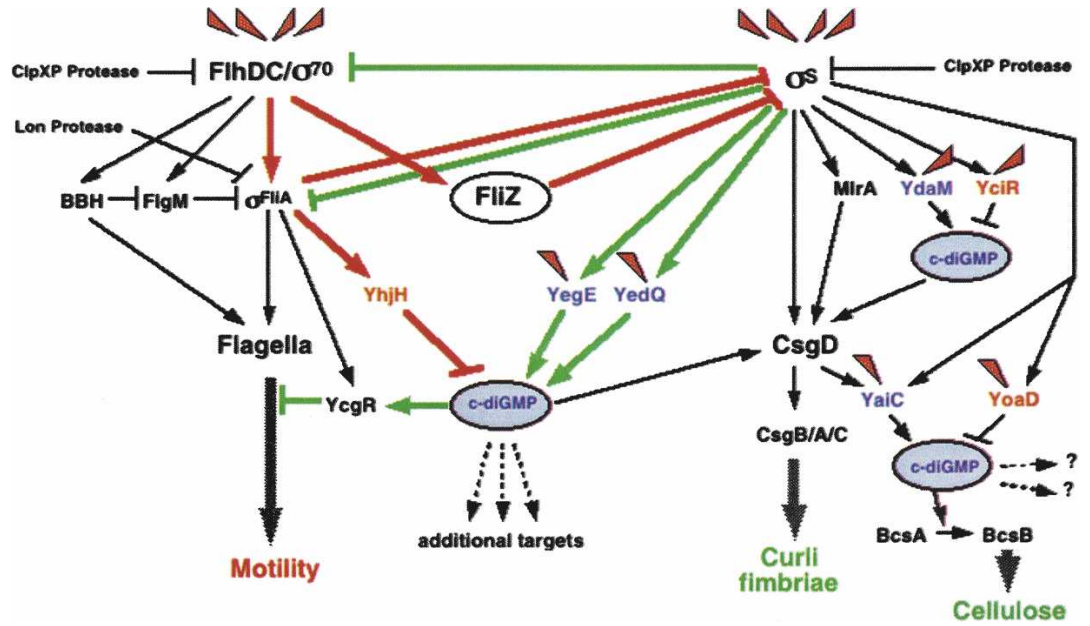


nential growth phase (Lange and Hengge-Aronis 1994), FliZ transiently gives motility (and therefore a foraging strategyl priority over the general stress response-as long as flagellar gene expression keeps going. FliZ acts as a timing factor for the expression of certain $\sigma^{\mathrm{s}}$-controlled genes, including those involved in curli expression (also thereby antagonizing $\mathrm{Crl}$ ), as illustrated by the mlrA and ydaM genes. Their timing of activation is precisely controlled by FliZ (Fig. 2) and by Crl (Supplemental Fig. S3). MlrA is most strongly affected and exhibits the latest induction among the three essential activators for $\operatorname{csg} D$ transcription (the other ones being $\sigma^{\mathrm{s}}$ and YdaM), and therefore can act as a timing device in the expression of the curli regulator CsgD.

Finally, a fliZ mutation resulted in premature expression of various additional $\sigma^{\mathrm{s}}$-dependent genes, which include two EAL genes (actually the majority of GGDEF/ EAL genes is under $\sigma^{\mathrm{S}}$ control) (our unpublished data). If the encoded c-di-GMP phosphodiesterases become active under these conditions, this would explain why a fliZ mutation can partially suppress the nonmotility phenotype of the yhjH mutant (Girgis et al. 2007; also observed by us).

Switching from motility to adhesion requires down-regulation of flagellar gene expression and proteolysis of the flagellar master regulator FlhDC

With FliZ giving priority to motility, down-regulation of the flagellar system is required to allow the activation of $\sigma^{\mathrm{S}}$-dependent genes, including the control cascade for the expression of the adhesive curli fimbriae. Consistently, the expression of the relevant regulatory genes flhDC, fliA, fliZ, and $y h j H$ ceases at an $\mathrm{OD}_{578}$ between 2 and 2.5 in LB-grown cells (actually in a temporal order that reflects the flagellar gene hierarchy) (Fig. 4). The curli regulator CsgD starts to accumulate, and expression of its target gene $\operatorname{csg} B$ begins shortly thereafter (at an $\mathrm{OD}_{578}$ of 2.8-3) (Fig. 6).

What is the triggering signal for this down-regulation of the entire flagellar system? Even though the c-di-GMP control system YegE/YhjH modulates motility (Fig. 3; see below), it is not involved in the down-regulation of flagellar gene expression (Fig. 4D). Also, mutations in regulatory systems known to be able to interfere with flhDC expression-i.e., the Rcs and BarA/UvrY twocomponent systems as well as LrhA (summarized in Prüß et al. 2006) — did not influence the post-exponential shut-down of flhDC expression (S. Busse, A. Kademci, and R.Hengge, unpubl.). Induction of $\sigma^{\mathrm{s}}$ itself and its successful competition with $\sigma^{70}$ (driven by ppGpp accumulation during entry into stationary phase (Lange et al. 1995; Jishage et al. 2002) may contribute to flhDC downregulation, as the latter is less pronounced in the rpoS mutant (Fig. 4B).

The observed down-regulation of flagellar gene expression and motility cannot be a consequence of repression of $f l h D C$ expression alone. In addition, the activator proteins of the flagellar system already present in the cell have to be inactivated. Dilution by further cell divisions would not be effective, as cells already approach stationary phase when these processes take place. Getting rid of proteins under such conditions often occurs by proteolysis (Jenal and Hengge-Aronis 2003). The transcriptional activators of the motility system-i.e., FlhDC and FliA - are known to be degraded by the ClpXP and Lon proteases, respectively (Tomoyasu et al. 2003; Barembruch and Hengge 2007). Here, we demonstrate that the Clp protease is indeed essential for the precisely timed reduction of the expression and therefore overall cellular activity of the c-di-GMP-degrading $\mathrm{PDE} \mathrm{YhjH}$, which has to be overcome to switch to curli induction (Figs. 5, 6). As $\mathrm{YhjH}$ is not degraded under these conditions (Supplemental Fig. S6), ClpXP-dependent proteolysis of FlhDC provides the basis for the curli-negative phenotype of the clpP mutant. That disappearance of FlhDC protein is absolutely necessary for curli induction, also finally provides a clear physiological rationale for FlhDC degradation.

Role of GGDEF/EAL proteins YegE, YedQ, YeaJ, and $\mathrm{YhjH}$ in the motility-to-adhesion switch

In E. coli, the YhjH protein is crucial for motility in post-exponential cells-i.e., either on nutrient-poor soft agar plates (Fig. 3A) or in liquid LB cultures beyond an $\mathrm{OD}_{578}$ of 3.5 -suggesting that it is the dominant c-di-GMP phosphodiesterase (Fig. 3B) under these conditions. This is in contrast to Salmonella, where a yhjH mutation has a minor effect on motility only (Frye et al. 2006; Ryjenkov et al. 2006; Simm et al. 2007). Reduced motility in the E. coli yhjH mutant depends on the presence of several GGDEF proteins, which act in an additive manner, with their relative contributions depending on the actual environmental conditions: YegE is most effective at $28^{\circ} \mathrm{C}$, YeaJ is the major player at $37^{\circ} \mathrm{C}$ (where curli are not expressed), and YedQ plays a minor role at both temperatures (Fig. 3; Supplemental S4). YegE contains a GGDEF domain as well as a degenerate EAL domain; YeaJ and YedQ are GGDEF-only proteins. All three proteins contain intact GGDEF motifs, representing the enzymatically active site (A-site) of diguanylate cyclases (Chan et al. 2004), as well as an intact I-site, which is an allosteric c-di-GMP-binding site imposing product inhibition on these enzymes (Chan et al. 2004; Christen et al. 2006). Taken together, this indicates that YegE, YeaJ, and YedQ are the di-guanylate cyclases that contribute to variable extents to a common pool of c-di-GMP, which can be degraded by $\mathrm{YhjH}$.

By binding to the effector protein YcgR, this c-di-GMP reduces motility (Ryjenkov et al. 2006; Christen et al. 2007). Mutations in all these factors do not affect the expression of the flagellar genes (Fig. 4D), which means that the effector protein YcgR probably interacts with a component of the flagellar basal body and thereby interferes with motor function directly. A similar conclusion was drawn for the activity of DgrA, a c-di-GMP-binding protein and homolog of YcgR, which in Caulobacter crescentus inhibits motility of swarmer cells (Christen et al. 2007). Consistent with a direct effect on motor 
function, a $y h j H$ mutant has an altered flagellar rotation pattern (Girgis et al. 2007). Taken together, inhibition of motility during entry into stationary phase occurs at all possible levels: Besides a shut-down of flagellar gene expression, which also depends on proteolytic elimination of FlhDC, activity of existing flagella is reduced by the c-di-GMP control module YegE + YedQ + YeaJ/YcgR.

In addition, the antagonists $\mathrm{YegE}$ and $\mathrm{YhjH}$ are key factors for inversely coordinating motility with curli expression. YegE is required for normal curli expression (Fig. 6A). Also, in Salmonella, YegE (STM2123) plays a positive role in curli formation, which, however, has not been clarified further (Kader et al. 2006). YhjH, on the other hand, is a clear inhibitor of curli expression (Fig. 6A). Interestingly, YegE/YhjH-controlled c-di-GMP affects motility via YcgR, but this effector is not involved in the control of curli expression (Fig. 6B). That c-diGMP controlled by $\mathrm{YegE} / \mathrm{YhjH}$ can act via different effector components suggests that this c-di-GMP is freely diffusible. The target of the $\mathrm{YegE} / \mathrm{YhjH}$ system in curli control is the transcription of the $\operatorname{csg} D$ gene (Fig. 6; Supplemental Figs. S7, S8). Taken together, FlhDC-dependent expression of $\mathrm{YhjH}$ in combination with $\sigma^{\mathrm{S}}$-dependent induction of its main antagonist YegE generates a switch mechanism based on titration of antagonistic enzymes, which functions as a checkpoint system that "unlocks" $\operatorname{csg} D$ and therefore curli expression only after the shut-down of flagellar gene expression during entry into stationary phase has occurred.

At the same time, another DGC/PDE pair, YdaM/ YciR, acts strongly and highly specifically on $\operatorname{csg} D$ transcription only (Fig. 6C; Weber et al. 2006), but does not affect motility (Fig. 3; Supplemental Fig. S5). At the moment, it is an open question how the two c-di-GMP control systems coordinate their activities at the $\operatorname{csg} D$ promoter, but our data suggest that the YdaM/YciR system does not contribute to the overall cellular c-di-GMP level (as YegE/YhjH does), but may act in a more local manner onto the $\operatorname{csg} D$ promoter region only. Sequestration of various DGC/PDE systems has previously been suggested based on the existance of sometimes several dozens of GGDEF/EAL proteins in single species (E. coli has 29 such proteins) (Jenal and Malone 2006; Kader et al. 2006; Ryan et al. 2006; Weber et al. 2006) and is certainly an issue that has to be studied more closely in the future.

\section{Conclusions and perspectives}

In the present study, we characterized the complex switch mechanism that controls the transition from motility-based foraging to the general stress response and curli-based adhesion that operates in E. coli during entry into stationary phase. Overall, this mechanism relies on mutual interference of two transcription factor cascades, which integrates transcription, proteolysis, and enzymatic activities. The switch operates on two clearly distinct hierarchical levels (Fig. 7). (1) At the top level, the activities of the unstable master regulators FlhDC and $\sigma^{\mathrm{s}}$ land therefore the entire motility and general stress response systems) are inversely controlled by $\sigma$ factor com- petition for core RNAP $\left(\sigma^{70}, \sigma^{\mathrm{F}}\right.$, and $\left.\sigma^{\mathrm{S}}\right)$ and by the FlhDC-dependent FliZ inhibiting $\sigma^{\mathrm{S}}$ activity. (2) At a lower level, the second messenger c-di-GMP, which specifically reduces flagellar activity and stimulates the expression of the adhesive curli fimbriae activator CsgD, is balanced by FlhDC-controlled degradation (via $\mathrm{YhjH}$ ) and $\sigma^{\mathrm{S}}$-controlled production (via YegE, YedQ).

In this system, shutting down flagellar gene expression is not just a strategy to avoid wasting energy for making new flagella that are no longer needed in cells that hardly grow any longer. Rather, it is part of an exquisitely balanced switching mechanism in which precisely timed inactivation of distinct genes under flagellar control (i.e., fli $Z$ and $y h j H)$, which also requires proteolytic removal of existing FlhDC, is a strict prerequisite for turning on the general stress response including curli formation and adhesion.

An interesting question for further studies is the nature of the trigger that actually throws the FlhDC/motility to $\sigma^{\mathrm{S}} /$ adhesion switch. Is it just $\sigma^{\mathrm{s}}$ accumulation as cells experience increasing nutrient limitation? If so, ppGpp is likely to be an essential trigger (Lange et al. 1995; Jishage et al. 2002). Also, how is the motility/adhesion switch integrated with other regulatory circuits that influence various biofilm functions; e.g., the Rcs or Csr regulatory systems?

Finally, this switch has to be rapidly reversible-at the top level, ClpXP-dependent proteolysis of $\sigma^{\mathrm{S}}$ may be essential for reversibility and, in fact, rapid degradation of $\sigma^{\mathrm{S}}$ is completely re-established within a couple of minutes upon refeeding of a stationary phase culture (Pruteanu and Hengge-Aronis 2002). At the lower c-di-GMP-controlled level, reversibility would be achieved by $\mathrm{YhjH}$ not being degraded, but rather outcompeted by various DGCs when cells enter into stationary phase. Whether the DGC/PDE-dependent c-di-GMP balance switches toward motility or toward curli expression (and other CsgD-dependent biofilm-associated functions) should depend not only on relative expression of the DGCs and PDEs involved, but also on the activities of their not yet characterized sensory input domains.

\section{Materials and methods}

\section{Bacterial strains and growth conditions}

All strains used in this study are derivatives of the E. coli $\mathrm{K}-12$ strains W3110 (Hayashi et al. 2006) or MC4100 (Casadaban 1976). All strains carry a $\Delta l a c$ deletion. The construction of mutant alleles, plasmids, and single-copy lacZ reporter fusions are described in detail in the Supplemental Material (including relevant references).

Cells were grown in LB medium (Miller 1972) at $28^{\circ} \mathrm{C}$ under aeration if not otherwise indicated (as this is the appropriate temperature for curli expression). Antibiotics and IPTG were added as detailed in the Supplemental Material. Growth was monitored by measuring the optical density at $578 \mathrm{~nm}\left(\mathrm{OD}_{578}\right)$.

\section{SDS page and immunoblot analysis}

Sample preparation for SDS-PAGE and immunoblot analysis were performed as described previously (Lange and Hengge-Aro- 
nis 1994). Five micrograms or $10 \mu \mathrm{g}$ of cellular protein were applied per lane. Polyclonal sera against $\sigma^{\mathrm{s}}, \mathrm{CsgD}$, and $\mathrm{YhjH}$ (custom-made by Pineda-Antikörper-Service), a goat anti-rabbit IgG alkaline phosphatase conjugate (Sigma), and a chromogenic substrate (BCIP/NBT; Boehringer Mannheim) were used.

\section{Northern blot analysis}

For RNA preparation and Northern blot analysis, cells were grown in $\mathrm{LB}$ medium at $28^{\circ} \mathrm{C}$ and harvested at an $\mathrm{OD}_{578}$ of 4.0 . The procedure and materials used were exactly as described before (Weber et al. 2006).

\section{c-di-GMP phosphodiesterase activity}

Phosphodiesterase activity of purified (see above) C-terminally His6-tagged $\mathrm{YhjH}$ and $\mathrm{YhjH}^{\mathrm{E} 48 \mathrm{~A}}(0.5 \mu \mathrm{M})$ was determined using radiolabeled c-di-GMP prepared with the purified di-guanylate cyclase PleD* exactly as described (Weber et al. 2006).

\section{Determination of $\beta$-galactosidase activity}

$\beta$-galactosidase activity was assayed by use of $o$-nitrophenyl- $\beta$ D-galactopyranoside (ONPG) as a substrate and is reported as micromoles of $o$-nitrophenol per minute per milligram of cellular protein (Miller 1972). Experiments showing the expression of lacZ fusions along the entire growth cycle were done at least twice, and a representative experiment is shown.

\section{Bacterial motility assay}

Motility was tested on swim plates containing $0.5 \%$ bacto-tryptone, $0.5 \% \mathrm{NaCl}$, and $0.3 \%$ agar. Three microliters of overnight cultures (adjusted to an $\mathrm{OD}_{578}$ of 4.0 in their own supernatant) were inocculated into the swim plates, and cells were allowed to grow and swim for $3-5 \mathrm{~h}$ at the temperature indicated.

\section{Acknowledgments}

Financial support was provided by the Deutsche Forschungsgemeinschaft (He 1556/13-1), the Dr. Hans-Messner-Stiftung, and the Fonds der Chemischen Industrie.

\section{References}

Adler, J. and Templeton, B. 1967. The effect of environmental conditions on the motility of Escherichia coli. J. Gen. Microbiol. 46: 175-184.

Aldrigde, P.D., Karlinsey, J.E., Aldridge, C., Birchall, C., Thompson, D., Yagasaki, J., and Hughes, K.T. 2006. The flagellarspecific transcription factor, $\sigma^{28}$, is the type III secretion chaperone for the flagellar-specific anti- $\sigma^{28}$ factor FlgM. Genes \& Dev. 20: 2315-2326.

Amsler, C.D., Cho, M., and Matsumura, P. 1993. Multiple factors underlying the maximum motility of Escherichia coli as cultures enter post-exponential growth. I. Bacteriol. 175: 6238-6244.

Barembruch, C. and Hengge, R. 2007. Cellular levels and activity of the flagellar $\sigma$ factor FliA of Escherichia coli are controlled by FlgM-modulated proteolysis. Mol. Microbiol. 65: 76-89.

Barker, C.S., Prüss, B.M., and Matsumura, P. 2004. Increased motility of Escherichia coli by insertion sequence element integration into the regulatory region of the $f 1 \mathrm{hD}$ operon. $J$. Bacteriol. 186: 7529-7537.
Barnhart, M.M. and Chapman, M.R. 2006. Curli biogenesis and function. Annu. Rev. Microbiol. 60: 131-147.

Benach, J., Swaminathan, S.S., Tamayo, R., Handelman, S.K., Folta-Stogniew, E., Ramos, J.E., Forouhar, F., Neely, H., Seetharaman, J., Camilli, A., et al. 2007. The structural basis of cyclic diguanylate signal transduction by PilZ domains. EMBO J. 26: 5153-5166.

Bokranz, W., Wang, X., Tschape, H., and Römling, U. 2005. Expression of cellulose and curli fimbriae by Escherichia coli isolated from the gastrointestinal tract. J. Med. Microbiol. 54: 1171-1182.

Bougdour, A., Lelong, C., and Geiselmann, J. 2004. Crl, a low temperature-induced protein in Escherichia coli that binds directly to the stationary phase $\sigma$ subunit of RNA polymerase. J. Biol. Chem. 279: 19540-19550.

Brombacher, E., Baratto, A., Dorel, C., and Landini, P. 2006. Gene expression regulation by the curli activtor CsgD protein: Modulation of cellulose biosynthesis and control of negative determinants for microbial adhesion. J. Bacteriol. 188: 2027-2037.

Brown, P.K., Dozois, C.M., Nickerson, C.A., Zuppardo, A., Terlonge, J., and Curtiss III, R. 2001. MlrA, a novel regulator of curli (Agf) and extracellular matrix synthesis by Escherichia coli and Salmonella enterica serovar typhimurium. Mol. Microbiol. 41: 349-363.

Casadaban, M.J. 1976. Transposition and fusion of the lac genes to selected promoters in Escherichia coli using bacteriophage $\lambda$ and $\mu$. J. Mol. Biol. 104: 541-555.

Chan, C., Paul, R., Samoray, D., Amiot, N., Giese, B., Jenal, U., and Schirmer, T. 2004. Structural basis of activity and allosteric control of diguanylate cyclase. Proc. Natl. Acad. Sci. 101: 17084-17089.

Chilcott, G.S. and Hughes, K.T. 2000. Coupling of flagellar gene expression to flagellar assembly in Salmonella enterica serovar typhimurium and Escherichia coli. Microbiol. Mol. Biol. Rev. 64: 694-708.

Christen, B., Christen, M., Paul, R., Schmid, F., Folcher, M., Jenoe, P., Meuwly, M., and Jenal, U. 2006. Allosteric control of cyclic di-GMP signaling. J. Biol. Chem. 281: 32015-32024.

Christen, M., Christen, B., Allan, M.G., Folcher, M., Jenö, P., Grzesiek, S., and Jenal, U. 2007. DgrA is a member of a new family of cyclic diguanosine monophosphate receptors and controls flagellar motor function in Caulobacter crescentus. Proc. Natl. Acad. Sci. 104: 4112-4117.

Frye, J., Karlinsey, J.E., Felise, H.R., Marzolf, B., Dowidar, N., McClelland, M., and Hughes, K.T. 2006. Identification of new flagellar genes of Salmonella enterica serovar typhimurium. J. Bacteriol. 188: 2233-2243.

Gerstel, U., Park, C., and Römling, U. 2003. Complex regulation of $\operatorname{csg} D$ promoter activity by global regulatory proteins. Mol. Microbiol. 49: 639-654.

Girgis, H.S., Liu, Y., Ryu, W.S., and Tavazoie, S. 2007. A comprehensive genetic characterization of bacterial motility. PLoS Genet. 3: e154. doi: 10.1371/journal.pgen.0030154.

Hall-Stoodley, L.K., Costerton, J.W., and Stoodley, P. 2004. Bacterial biofilms: From the natural environment to infectious diseases. Nat. Rev. Microbiol. 2: 95-108.

Hayashi, K., Morooka, N., Yamamoto, Y., Fujita, K., Isono, K., Choi, S., Ohtsubo, E., Baba, T., Wanner, B.L., Mori, H., and Horiuchi, T. 2006. Highly accurate genome sequences of Escherichia coli K-12 strains MG1655 and W3110. Mol. Syst. Biol. 2: 2006.0007. doi: 10.1038/msb4100049.

Hengge-Aronis, R. 2000. The general stress response in Escherichia coli. In Bacterial stress responses (eds. G. Storz and R. Hengge-Aronis), pp. 161-178. ASM Press, Washington, DC.

Hengge-Aronis, R. 2002. Signal transduction and regulatory 
mechanisms involved in control of the $\sigma^{\mathrm{S}}$ subunit of RNA polymerase in Escherichia coli. Microbiol. Mol. Biol. Rev. 66: 373-395.

Jenal, U. and Hengge-Aronis, R. 2003. Regulation by proteolysis in bacterial cells. Curr. Opin. Microbiol. 6: 163-172.

Jenal, U. and Malone, J. 2006. Mechanisms of cyclic-di-GMP signaling in bacteria. Annu. Rev. Genet. 40: 385-407.

Jishage, M.L. and Ishihama, A. 1997. Variation in RNA polymerase $\sigma$ subunit composition within different stocks of Escherichia coli W3110. J. Bacteriol. 179: 959-963.

Jishage, M., Kvint, K., Shingler, V., and Nyström, T. 2002. Regulation of $\sigma$ factor competition by the alarmone ppGpp. Genes \& Dev. 16: 1260-1270.

Kader, A., Simm, R., Gerstel, U., Morr, M., and Römling, U. 2006. Hierarchical involvement of various GGDEF domain proteins in rdar morphotype development of Salmonella enterica serovar typhimurium. Mol. Microbiol. 60: 602-616.

Karlinsey, J.E., Tanaka, S., Bettenworth, B., Yamaguchi, S., Boos, W., Aizawa, S.-I., and Hughes, K.T. 2000. Completion of the hook-basal body complex of the Salmonella typhimurium flagellum is coupled to $\mathrm{FlgM}$ secretion and fliC transcription. Mol. Microbiol. 37: 1220-1231.

Klausen, M., Gjermansen, M., Kreft, J.U., and Tolker-Nielsen, T. 2006. Dynamics of development and dispersal in sessile microbial communities: Examples form Pseudomonas aeruginosa and Pseudomonas putida model biofilms. FEMS Microbiol. Lett. 261: 1-11.

Ko, M. and Park, C. 2000. Two novel flagellar components and $\mathrm{H}-\mathrm{NS}$ are involved in the motor function of Escherichia coli. I. Mol. Biol. 303: 371-382.

Kutsukake, K., Ikebe, T., and Yamamoto, S. 1999. Two novel regulatory genes, fliT and fliZ, in the flagellar regulon of Salmonella. Genes Genet. Syst. 74: 287-292.

Lange, R. and Hengge-Aronis, R. 1994. The cellular concentration of the $\sigma^{\mathrm{S}}$ subunit of RNA-polymerase in Escherichia coli is controlled at the levels of transcription, translation and protein stability. Genes \& Dev. 8: 1600-1612.

Lange, R., Fischer, D., and Hengge-Aronis, R. 1995. Identification of transcriptional start sites and the role of ppGpp in the expression of rpoS, the structural gene for the $\sigma^{\mathrm{s}}$ subunit of RNA-polymerase in Escherichia coli. J. Bacteriol. 177: 46764680.

Liu, X. and Matsumura, P. 1994. The FlhD/FlhC complex, a transcriptional activator of the Escherichia coli flagellar class II operons. J. Bacteriol. 176: 7345-7351.

Miller, J.H. 1972. Experiments in molecular genetics. Cold Spring Harbor Laboratory, Cold Spring Harbor, NY.

Olsén, A., Jonsson, A., and Normark, S. 1989. Fibronectin binding mediated by a novel class of surface organelles on Escherichia coli. Nature 338: 652-655.

O'Toole, G.A., Kaplan, H.B., and Kolter, R. 2000. Biofilm formation as microbial development. Annu. Rev. Microbiol. 54: 49-79.

Prüß, B.M., Besemann, C., Denton, A., and Wolfe, A.J. 2006. A complex transcription network controls the early stages of biofilm development in Escherichia coli. J. Bacteriol. 188: 3731-3739.

Pruteanu, M. and Hengge-Aronis, R. 2002. The cellular level of the recognition factor RssB is rate-limiting for $\sigma^{\mathrm{S}}$ proteolysis: Implications for RssB regulation and signal transduction in $\sigma^{\mathrm{s}}$ turnover in Escherichia coli. Mol. Microbiol. 45: 1701-1714.

Römling, U., Rohde, M., Olsén, A., Normark, S., and Reinköster, J. 2000. AgfD, the checkpoint of multicellular and aggregative behaviour in Salmonella typhimurium regulates at least two independent pathways. Mol. Microbiol. 36: 10-23.

Römling, U., Gomelsky, M., and Galperin, M.Y. 2005. C-di-
GMP: The dawning of a novel bacterial signalling system Mol. Microbiol. 57: 629-639.

Ryan, R.P., Fouhy, Y., Lucey, J.F., and Dow, J.M. 2006. Cyclic di-GMP signaling in bacteria: Recent advances and new puzzles. J. Bacteriol. 188: 8327-8334.

Rychlik, I., Martin, G., Methner, U., Lovell, M., Cardova, L., Sebkova, A., Sevcik, M., Damborsky, J., and Barrow, P.A. 2002. Identification of Salmonella enterica serovar Typhimurium genes associated with growth suppression in stationary-phase nutrient broth cultures and in the chicken intestine. Arch. Microbiol. 178: 411-420.

Ryjenkov, D.A., Simm, R., Römling, U., and Gomelsky, M 2006. The PilZ domain is a receptor for the second messenger c-di-GMP: The PilZ protein YcgR controls motility in enterobacteria. J. Biol. Chem. 281: 30310-30314.

Simm, R., Lusch, A., Kader, A., Andersson, M., and Römling, U. 2007. Role of EAL-containing proteins in multicellular behavior of Salmonella enterica serovar typhimurium. J. Bacteriol. 189: 3613-3623.

Subramanya, H.S., Arciszweska, L.K., Baker, R.A., Bird, L.E., Sherratt, D.J., and Wiglex, D.B. 1997. Crystal structure of the site-specific recombinase, XerD. EMBO J. 16: 5178-5187.

Tamayo, R., Pratt, J.T., and Camilli, A. 2007. Roles of cyclic diguanylate in the regulation of bacterial pathogenesis. Annu. Rev. Microbiol. 61: 131-148.

Tomoyasu, T., Takaya, A., Isogai, E., and Yamamoto, T. 2003. Turnover of FlhD and FlhC, master regulator proteins for Salmonella flagellum biogenesis, by the ATP-dependent ClpXP protease. Mol. Microbiol. 48: 443-452.

Typas, A., Barembruch, C., and Hengge, R. 2007a. Stationary phase reorganisation of the $E$. coli transcription machinery by $\mathrm{Crl}$ protein, a fine-tuner of $\sigma^{\mathrm{S}}$ activity and levels. $E M B O$ J. 26: 1569-1578.

Typas, A., Becker, G., and Hengge, R. 2007b. The molecular basis of selective promoter activation by the $\sigma^{\mathrm{S}}$ subunit of RNA polymerase. Mol. Microbiol. 63: 1296-1306.

Wang, S., Fleming, R.T., Westbrook, E.M., Matsumura, P., and McKay, D.B. 2006. Structure of the Escherichia coli FlhDC complex, a prokaryotic heteromeric regulator of transcription. J. Mol. Biol. 355: 798-808.

Weber, H., Polen, T., Heuveling, J., Wendisch, V., and Hengge, R. 2005. Genome-wide analysis of the general stress response network in Escherichia coli: $\sigma^{\mathrm{s}}$-dependent genes, promoters and $\sigma$ factor selectivity. J. Bacteriol. 187: 1591-1603.

Weber, H., Pesavento, C., Possling, A., Tischendorf, G., and Hengge, R. 2006. Cyclic-di-GMP-mediated signaling within the $\sigma^{\mathrm{s}}$ network of Escherichia coli. Mol. Microbiol. 62: 10141034.

Zhao, K., Liu, M., and Burgess, R.R. 2007. Adaptation in bacterial flagellar and motility systems: From regulon members to "foraging"-like behavior in E. coli. Nucleic Acids Res. 35: $4441-4452$. 


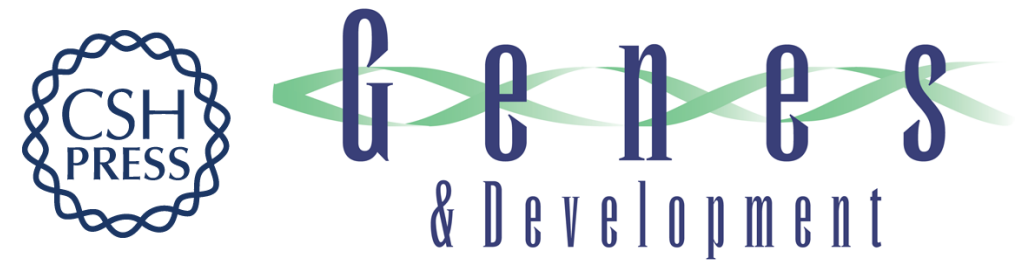

\section{Inverse regulatory coordination of motility and curli-mediated adhesion in Escherichia coli}

Christina Pesavento, Gisela Becker, Nicole Sommerfeldt, et al.

Genes Dev. 2008, 22:

Access the most recent version at doi:10.1101/gad.475808

Supplemental http://genesdev.cshlp.org/content/suppl/2008/08/14/22.17.2434.DC1
Material

References This article cites 53 articles, 22 of which can be accessed free at:

http://genesdev.cshlp.org/content/22/17/2434.full.html\#ref-list-1

License

Email Alerting

Receive free email alerts when new articles cite this article - sign up in the box at the top

Service

right corner of the article or click here.

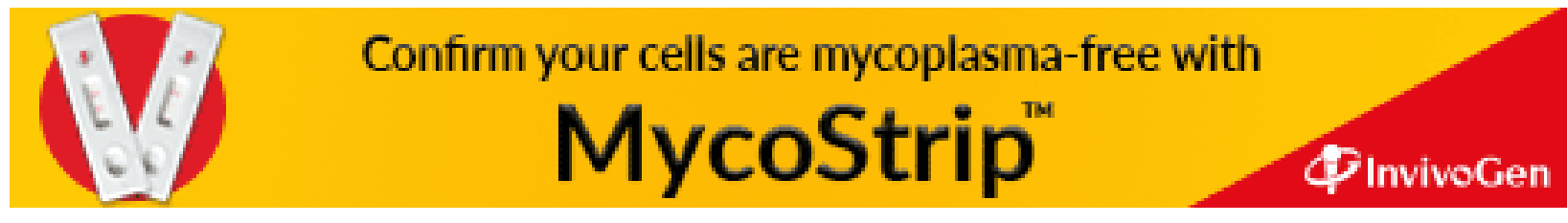

